Ferrata Storti Foundation

\title{
A gain-of-function RAC2 mutation is associated with bone marrow hypoplasia and an autosomal dominant form of severe combined immunodeficiency
}

\author{
Chantal Lagresle-Peyrou, ${ }^{1,2,3}$ Aurélien Olichon, ${ }^{4}$ Hanem Sadek, ${ }^{3}$ \\ Philippe Roche,${ }^{5}$ Claudine Tardy,${ }^{4}$ Cindy Da Silva, ${ }^{3}$ Alexandrine Garrigue, ${ }^{3}$ \\ Alain Fischer, ${ }^{2,6,7}$ Despina Moshous, ${ }^{6}$ Yves Collette, ${ }^{5}$ Capucine Picard,,${ }^{2,6,8,9}$ \\ Jean Laurent Casanova, ,,6,10,11,12 Isabelle André ${ }^{1,2}$ and Marina Cavazzana ${ }^{1,2,3}$
}

Haematologica 2021

Volume 106(2):404-411

${ }^{1}$ Laboratory of Human Lymphohematopoiesis, INSERM UMR 1163, Imagine Institute, F-75015 Paris, France; ${ }^{2}$ Paris Descartes University - Sorbonne Paris Cité, Imagine Institute UMR1163, F-75015 Paris, France; ${ }^{3}$ Biotherapy Clinical Investigation Center, Groupe Hospitalier Universitaire Ouest, Assistance Publique-Hôpitaux de Paris, INSERM CIC 1416, F-75015 Paris, France; ${ }^{4}$ Cancer Research Center of Toulouse, CRCT, University of Toulouse, UPS, INSERM U1037, F-31037 Toulouse, France; ${ }^{5}$ Marseille Cancer Research Center, CRCM, Aix Marseille University, Institut Paoli-Calmettes, CNRS, INSERM, Team ISCB, F-13273 Marseille, France; ${ }^{6}$ Department of Pediatric Immunology, Hematology and Rheumatology, Necker-Enfants Malades University Hospital, APHP, F75015 Paris, France; ${ }^{7}$ College de France, F-75231 Paris, France; ${ }^{8}$ Study Center for Primary Immunodeficiencies, Assistance Publique-Hôpitaux de Paris (AP-HP), Necker-

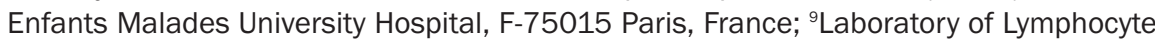
Activation and Susceptibility to EBV, INSERM UMR 1163, Imagine Institute, F-75015 Paris, France; ${ }^{10}$ Laboratory of Human Genetics of Infectious Diseases, Necker Branch INSERM UMR 1163, Imagine Institute, F-75015 Paris, France; ${ }^{11}$ St. Giles Laboratory of Human Genetics of Infectious Diseases, Rockefeller Branch, Rockefeller University, New York, NY, USA and ${ }^{12}$ Howard Hughes Medical Institute, New York, NY, USA

\section{ABSTRACT}

vere combined immunodeficiencies (SCID) constitute a heterogeneous S group of life-threatening genetic disorders that typically present in the first year of life. They are defined by the absence of autologous $T$ cells

\section{Correspondence:}

MARINA CAVAZZANA

m.cavazzana@aphp.fr

CHANTAL LAGRESLE-PEYRO

chantal.lagresle@inserm.fr

Received: June 20, 2019.

Accepted: January 8, 2020.

Pre-published: January 9, 2020.

https://doi.org/10.3324/haematol.2019.230250

(C)2021 Ferrata Storti Foundation

Material published in Haematologica is covered by copyright. All rights are reserved to the Ferrata Storti Foundation. Use of published material is allowed under the following terms and conditions:

https://creativecommons.org/licenses/by-nc/4.0/legalcode. Copies of published material are allowed for personal or internal use. Sharing published material for non-commercial purposes is subject to the following conditions:

https://creativecommons.org/licenses/by-nc/4.0/legalcode, sect. 3. Reproducing and sharing published material for commercial purposes is not allowed without permission in writing from the publisher. and the presence of an intrinsic or extrinsic defect in the B-cell compartment. In three newborns presenting with frequent infections and profound leukopenia, we identified a private, heterozygous mutation in the RAC2 gene (p.G12R). This mutation was de novo in the index case, who had been cured by hematopoietic stem cell transplantation but had transmitted the mutation to her sick daughter. Biochemical assays showed that the mutation was associated with a gain of function. The results of in vitro differentiation assays showed that RAC2 is essential for the survival and differentiation of hematopoietic stem/progenitor cells. Therefore, screening for RAC2 gain-offunction mutations should be considered in patients with a SCID phenotype and who lack a molecular diagnosis.

\section{Introduction}

Severe combined immunodeficiencies (SCID) are inherited primary immunodeficiencies characterized by a profound impairment of T-cell development and an intrinsic or functional defect in the B-cell compartment. Life-threatening SCID are the most severe primary immunodeficiencies; in the absence of treatment, SCID lead to death within the first year of life. The only curative treatments are allogeneic hematopoietic stem cell transplantation (HSCT) or, for two types of SCID, autologous gene-modified HSCT. ${ }^{1}$ At present, all the SCID with a known molecular defect have an autosomal recessive pattern of inheritance or, in one case, Xlinked inheritance. Less than 5\% of SCID patients do not have a molecular diagnosis. ${ }^{2,3}$

Reticular dysgenesis (RD) is the SCID form with the earlier clinical presentation (i.e., a few days after birth), due to the absence of both neutrophils and T cells. In 
addition to the hematopoietic defect, patients with $\mathrm{RD}$ have bilateral sensorineural deafness. ${ }^{4}$ We and others have previously demonstrated that bi-allelic mutations in the gene coding for adenylate kinase 2 (AK2) are involved in the RD phenotype by disrupting the ATP production required to sustain the survival and differentiation of hematopoietic stem/progenitor cells (HSPC). The high AK2 expression levels in the inner ear may also account for the sensorineural hearing loss observed in patients with $\mathrm{RD}^{5-7}$

We have identified an autosomal dominant (AD) missense mutation in the RAC2 gene (coding for Ras-related C3 botulinum toxin substrate 2 [RAC2]) in three SCID patients whose clinical presentation overlaps with the $\mathrm{RD}$ SCID but who lack AK2 mutations and deafness. RAC2 belongs to the Rac subfamily of RHO small GTPases. In the inactive GDP-bound state, RAC2 is located in the cytosol. Upon stimulation and activation by guanine nucleotide exchange factors, the active RAC2-GTP-bound form translocates to the plasma membrane. There, active RAC2 protein triggers various signaling pathways until the GTP is hydrolyzed following binding to GTPase-activating proteins (GAP) ${ }^{8-10}$ Unlike the other members of the Rac subfamily (RAC1 and RAC3), RAC2 is mostly expressed on hematopoietic cells. ${ }^{11,12}$ Using biochemical and in vitro differentiation assays, we demonstrated that the RAC2 mutation was closely related to an impairment in cell differentiation capacity and defects in cellular and mitochondrial networks. Taken as a whole, our data demonstrate that a dominant gain-of-function (GOF) mutation in the GDP/GTP binding site of the RAC2 protein inhibits HSPC differentiation and leads to a severe $\mathrm{AD}$ form of SCID with a clinical presentation of RD.

\section{Methods}

\section{Patients and human cord blood samples}

The study was conducted in accordance with French legislation and the principles of the Declaration of Helsinki. Informed consent was obtained from the patients' parents or legal guardians, and the study protocol was approved by the regional independent ethics committee and the French Ministry of Research (DC 20142272/2015/ DC-2008-329). Primary fibroblasts were obtained from skin biopsies.

Human cord blood (CB) samples eligible for research purposes were obtained from the Cord Blood Bank at St Louis Hospital (Paris, France; authorization 2014/09/23). Mononuclear cells were isolated by density separation on Lymphoprep (Abcys). CD34 ${ }^{+}$ HSPC were sorted magnetically using the autoMACSpro separator, and cell purity was checked with a MACSQuant analyzer (Miltenyi Biotec) before transduction and culture (Online Supplementary Methods).

\section{Gene sequencing and gene expression in the patient's fibroblasts}

Genomic DNA was isolated by phenol/chloroform extraction from primary fibroblasts (from P1, P2 and P3) or peripheral blood mononuclear cells (PBMC, from P3's father). Whole-exome sequencing was performed with an Illumina TruSeq exome enrichment kit (Illumina), using 100 bp paired-end reads. Eightyfive percent of the target regions were observed with a coverage $>20 \times$. Variant calling was not based on a particular genetic model. The mutation was confirmed by Sanger sequencing. For western blot analyses, cells were lysed in Tris buffer (20 mM Tris, pH 7.9;
$300 \mathrm{mM} \mathrm{NaCl} ; 1 \%$ Nonidet P-40) supplemented with protease and phosphatase inhibitors. Cell extracts were separated by SDSPAGE, blotted, and stained with anti-RAC2 (ab154711, Abcam) or anti-GAPDH (SC-32233, Santa Cruz, CA, USA) antibodies. After staining with an HRP-conjugated secondary antibody, the immunoblot was developed and quantified in an Odyssey system (LiCor).

\section{RAC2 activation assays and immunoblotting with the HEK293T cell line}

The HEK293T cells were cultured overnight prior to transduction with the appropriate lentiviral supernatant (Online Supplementary Methods). After two days of culture, the cell pellets were frozen in liquid nitrogen. Levels of activated RAC2 were determined using the G-LISA ${ }^{\circledR}$ RAC Activation Assay Biochem KitTM (\#BK125, Cytoskeleton Inc.) according to the manufacturer's instructions, except that RAC2 monoclonal specific antibody (AT2G11, sc-517424 Santa Cruz Biotechnology, Inc.) and HRPconjugated anti-mouse antibody (\#1721011, Bio-Rad) were used to detect the amount of captured active RAC2 (details in the Online Supplementary Methods).

\section{Homology modeling}

Three-dimensional homology models were built for the G12R mutant of human RAC2 (Uniprot P15153) using MODELLER software (version 20, Webb and Sali, 2016, 2017). The crystal structure of wild-type (WT) human RAC2 (PDB code: 1DS6) was used as a template.

\section{Statistical analysis}

For all analyses, three or more independent experiments were performed. Data are reported as the mean \pm standard error of the mean (SEM). Two-tailed, unpaired $t$-tests were performed using Prism 4 software (GraphPad). $P<0.05$ was considered statistically significant.

\section{Results}

\section{A missense mutation (G12R) in the RAC2 GTPase protein leads to a severe combined immunodeficiencies phenotype}

A few members of our cohort of patients with a SCID phenotype do not yet have a molecular diagnosis. Three patients (Figure 1A) from two unrelated kindreds (P1 from one kindred, and mother and daughter P2 and P3 from another kindred) presented with all the immunological and clinical features of $\mathrm{RD}$ other than deafness. As observed in patients with $\mathrm{RD}$, the complete absence of neutrophils was responsible for the occurrence of severe infections (Table 1) earlier than is usually observed in other forms of SCID. ${ }^{3}$ The analysis of other blood cell lineages highlighted the absence of $\mathrm{T}$ and $\mathrm{B}$ lymphocytes and circulating monocytes. The T-cell receptor excision circle (TREC) level was very low $(<5$ copies/ $\mu \mathrm{L}$ in $\mathrm{P} 3$, the only patient tested, vs. $>34$ copies for a control), reflecting defective T-cell maturation. Conversely, the platelet count was normal and (in P3 only) the hemoglobin level was slightly below normal (Table 1). Overall, the peripheral cell count profile was in line with the severe hypoplastic content of the patients' bone marrow (BM) (Table 1 and Online Supplementary Table S1). HSCT performed soon after birth was successful in P1 (who was previously described as P4 by André-Schmutz et al. ${ }^{13}$ ) and P2, with full donor immune reconstitution, demonstrating that the 
A

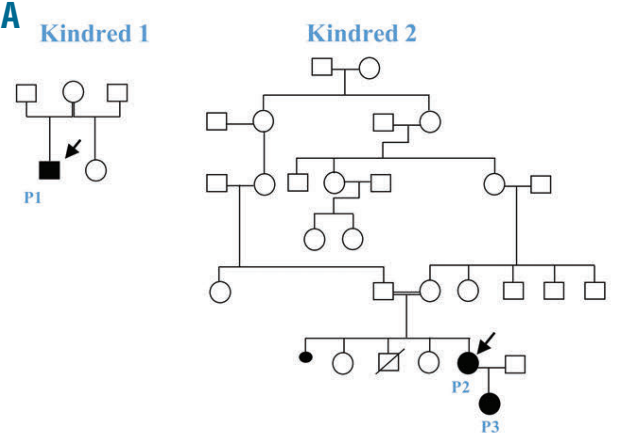

B

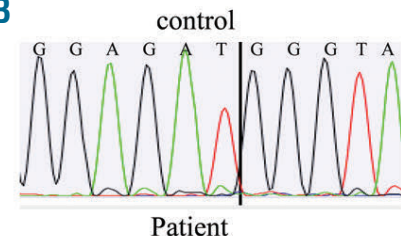

C

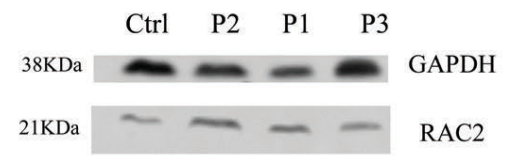

D

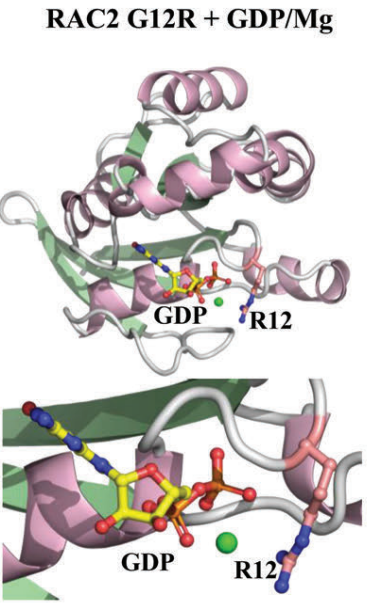

RAC2 G12R + GTP/Mg

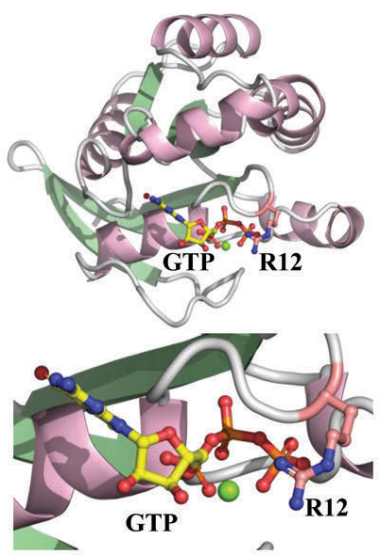

E

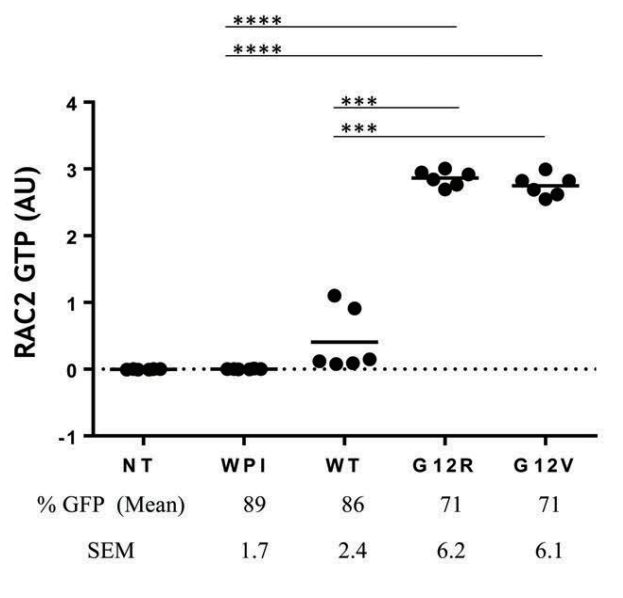

Figure 1. Identification and effects of the p.G12R RAC2 mutation on the GTPase activity of RAC2. (A) Pedigrees of the three patients from two unrelated kindred. Black boxes and black circles respectively represent the affected male (P1) and affected females (P2, P3). White boxes and circles respectively represent unaffected males and females. Arrows represent the probands and a double horizontal bar represents consanguinity. (B) A representative electropherogram of RAC2 DNA sequencing for control cells and patient cells, showing the c.34G>A mutation. (C) A representative immunoblot of RAC2 protein expression in lysates from control fibroblasts (Ctrl) and fibroblasts derived from the affected individuals (P1, P2, and P3). The loading control corresponds to GAPDH expression. (D) 3D models of the RAC2 G12R mutant. The structures are shown with GDP (left panel) or GTP (right panel) in the G1 binding pocket. For each model, a close-up view of the GDP/GTP binding pocket (dotted brown circle) is shown below the overall view. The figure was generated with the pymol program (www.pymol.org/). (E) HEK293T cells were either not transduced (NT, control) or transduced with a lentiviral empty vector (WPI) containing the wild-type (WT) form of RAC2 cDNA, the mutated form described here (G12R) or (as a positive control) the constitutively activated GTP-bound RAC2 form (G12V). Two days after transduction, cells were recovered for analysis using the G-LISA assay (15 $\mathrm{\mu g}$ of total protein per well) for the quantification of the GTP-bound RAC2 form (RAC2 GTP). The results come from three independent experiments, and the table below the graph represents the mean of the percentage of GFP expressing cells (GFP+) in the three independent experiments. $* * \star P<0.001$; $* * * * P<0.0001$.

inherited defect was intrinsic and not extrinsic (i.e., not micro-environmental) (Table 1). No other clinical symptoms have been reported so far for these patients (12 and 21 years after HSCT for P1 and P2, respectively), both of whom have achieved a good quality of life.

By performing whole-genome sequencing on the patients' fibroblasts (the only available cell source, since the patients underwent HSCT early in life), we identified a heterozygous missense mutation (c.34G $>$ A, p.G12R) in the RAC2 gene that was absent in P3's father, the only relative from whom we could obtain a DNA sample (Figure $1 \mathrm{~A}$ and B, and Online Supplementary Figure S1A). This variant (confirmed by Sanger sequencing) was not annotated in our in-house database ( $n=14,154$ samples in the cohort) or in the human Genome Aggregation Database, and was predicted to be deleterious by four different in silico prediction software tools, including Combined Annotation Dependent Depletion (Online Supplementary Figure S1B). The mutation was, therefore, considered to be diseasecausing. The presence of the same clinical phenotype and the same RAC2 G12R missense mutation in P2 and her daughter $\mathrm{P} 3$ highlighted an $\mathrm{AD}$ inheritance pattern.
The p.G12R missense mutation is located in the G1 box, a highly conserved guanine nucleotide binding region. ${ }^{14}$ It is noteworthy that this mutation differed from the loss-offunction (LOF) or gain-of-function (GOF) mutations (Online Supplementary Figure S1A) previously reported as being responsible for mild neutrophil defects and/or lymphopenia. ${ }^{15-20}$ Interestingly, the G12R missense mutation did not substantially alter RAC2 protein expression level in patient fibroblasts (Figure $1 \mathrm{C}$ and Online Supplementary Figure S1C).

\section{G12R mutation in the GDP/GTP-binding domain disrupts cell homeostasis}

In order to understand the mutation's functional impact, we generated a 3D homology model by using in silico models of WT RAC221 as a template. The structural models correspond to the RAC2 G12R mutant form with GDP bound (Figure 1D, left panel) or GTP bound (Figure 1D, right panel) state. In the GTP-bound RAC2 state, the bulky flexible arginine is in tight contact with the terminal phosphate group and may induce steric clashes that prevent GAP proteins from accessing the G1 binding pocket 
Table 1. Hematologic characteristics and outcomes for the three patients.

\begin{tabular}{|c|c|c|c|c|}
\hline $\begin{array}{l}\text { Patients } \\
\text { (age at presentation) }\end{array}$ & $\begin{array}{c}\text { P1 } \\
\text { (3 days) }\end{array}$ & $\begin{array}{c}\text { P2 } \\
\text { (10 days) }\end{array}$ & $\begin{array}{c}\text { P3 } \\
\text { (9 days) }\end{array}$ & $\begin{array}{l}\text { Age-matched } \\
\text { control value }\end{array}$ \\
\hline Infection at birth & Sepsis & $\begin{array}{l}\text { Colored amniotic fluid, } \\
\text { sepsis/pneumonia }\end{array}$ & $\begin{array}{l}\text { Sepsis/meningitis, } \\
\text { brain abscesses }\end{array}$ & \\
\hline White blood cells $\left(x 10^{9} / \mathrm{L}\right)$ & 0.6 & 0.3 & 0.5 & $7-18$ \\
\hline Lymphocytes (x109/L) & 0.4 & 0.1 & 0.5 & $3.4-7.6$ \\
\hline B lymphocytes (x10 $/ \mathrm{L})$ & 0.09 & 0.004 & 0 & $0.3-2$ \\
\hline T lymphocytes (x10 $/ \mathrm{L})$ & 0.24 & 0.07 & 0 & $2.5-5.5$ \\
\hline Monocytes $\left(x 10^{9} / \mathrm{L}\right)$ & 0.01 & $\mathrm{NE}$ & 0 & $0.1-1.1$ \\
\hline Neutrophils $\left(\mathrm{x} 10^{\circ} \mathrm{L}\right)$ & 0,2 & NE & 0 & $1.5-8.5$ \\
\hline Platelets $\left(\times 10^{9} / \mathrm{L}\right)$ & 248 & 220 & 429 & $175-500$ \\
\hline Hemoglobin $(\mathrm{g} / \mathrm{dL})$ & 18 & 13 & 10 & $12.5-16.6$ \\
\hline Bone marrow aspirate & Hypoplasia & Hypoplasia & Hypoplasia & \\
\hline HSCT (age at transplantation) & $1^{\text {st }}$ T-depleted HSCT (3M) & HSCT (2 M) & $1^{\text {st }}$ T-depleted HSCT (2 M) & \\
\hline \multirow[t]{7}{*}{ conditioning regimen } & Busulfan $8 \mathrm{mg} / \mathrm{kg}$ & Busulfan $8 \mathrm{mg} / \mathrm{kg}$ & Busulfan $3.6 \mathrm{mg} / \mathrm{kg}$ & \\
\hline & Cyclophosphamide $200 \mathrm{mg} / \mathrm{kg}$ & Cyclophosphamide 2,000 mg/kg & Fludarabine $160 \mathrm{mg} / \mathrm{m}^{2}$ & \\
\hline & ALS 25 mg/kg & & ALS 5 mg/kg & \\
\hline & $2^{\text {nd }}$ T-depleted HSCT (6M) & & $2^{\text {nd }}$ HSCT (3M) & \\
\hline & Busulfan $16 \mathrm{mg} / \mathrm{kg}$ & & Fludarabine $120 \mathrm{mg} / \mathrm{m}^{2}$ & \\
\hline & Cyclophosphamide $200 \mathrm{mg} / \mathrm{kg}$ & & ALS 5 mg/kg & \\
\hline & ALS 25 mg/kg & & & \\
\hline \multirow[t]{2}{*}{ Donor cells } & $1^{\text {st }} \mathrm{HSCT}: \mathrm{MMFD} / \mathrm{f}$ & MMFD/f & $1^{\text {st HSCT: MMFD/f }}$ & \\
\hline & $2^{\text {nd }}$ HSCT: MMFD/f & & $2^{\text {nd }}$ HSCT: MMFD/f & \\
\hline Outcome & AW & GvHD resolved at D120; AW & $\begin{array}{c}\text { Graft failure; } \\
\text { death (5.5 M post-HSCT) }\end{array}$ & \\
\hline
\end{tabular}

BM: bone marrow; HSCT: hematopoietic stem cell transplantation; M: months; ALS: antilymphocyte serum; MMFD/f: mismatched family donor/father; GvHD: graft-versus-host disease; A/W: alive and well (full donor immune reconstitution, and no other symptoms/diseases); NE: not evaluated.

(Figure 1D) and thus may impair the GTP hydrolysis rate, as previously demonstrated for other small GTPases. ${ }^{22}$

To test this model biochemically, we quantified the active GTP-bound RAC2 form (RAC2 GTP) in extracts of HEK293T cells not expressing RAC2 at the basal level. The cells were transduced with an empty lentiviral backbone (WPI) with green fluorescent protein (GFP) as a tracker or the vector containing either the WT form of $R A C 2$ cDNA, the mutated form described here (G12R) or (as positive control) the constitutively activated GTPbound form (G12V). ${ }^{23}$ A high level of the active GTPbound RAC2 state was observed for both G12V and G12R (Figure 1E), demonstrating that substitution by arginine at position 12 increases the level of active RAC2 protein; hence, the G12R mutation is associated with GOF.

To determine how the expression of a constitutively active form of RAC2 impacts cell division and survival, we used in vivo live cell imaging to measure the growth kinetics of primary fibroblasts. The proliferation rate (the change in the percentage of confluence) was significantly slower for P3's cells than for control cells throughout the culture period (Figure 2A, upper panel). Conversely, the proportion of dead cells (measured using Cytotox green reagent) was significantly higher for the P3 experiment (Figure 2A, lower panel). These results demonstrated that, in vitro, G12R-mutated cells show impaired proliferative capacity and a high mortality rate. To further characterize the cellular defects, we used non-invasive holotomographic live cell imaging to observe the cells' biological features and organelles. When compared with control fibroblasts,
P3's cells displayed very slow cell dynamics over the 12 hour culture period (Online Supplementary Movie V1); this was correlated with the low proliferative response. In P3's fibroblasts, the nuclear membrane was highly segmented, and the mitochondrial network was disrupted (Figure $2 \mathrm{~B}$ and Online Supplementary Figure S2A). The alteration of the mitochondrial network was confirmed by confocal microscopy (Online Supplementary Figure S2B); the proportion of fragmented mitochondrial networks was higher in P3's fibroblasts (35\%) than in control fibroblasts (10\%).

\section{G12R mutation inhibits hematopoietic stem/progenitor cell proliferation and differentiation}

To understand the impact of constitutive RAC2 activation on hematopoiesis and given the absence of patient BM samples, CD34+ human cord blood HSPC were transduced with the WPI, WT, G12R or G12V RAC2 cDNAs (with GFP as a tracker) and then cultured with cytokines for 7 days (Figure 3A). The expression of constitutively activated RAC2 forms (G12R and G12V) led to the disappearance of $\mathrm{GFP}^{+}$transduced cells within 4 days. The $\mathrm{GFP}^{+}$ cell ability to generate reactive hydroxyl radical (a common reactive oxygen species [ROS]) was significantly impaired in the G12R and G12V conditions (quantified as the proportion of "ROS low" cells) (Figure 3B). It is noteworthy that the addition of an ROS inducer was not associated with an elevation in ROS production (data not shown). To analyze this phenomenon in more detail, we evaluated the mitochondrial membrane potential in GFP transduced cells. In the G12R and G12V experiments, 
A
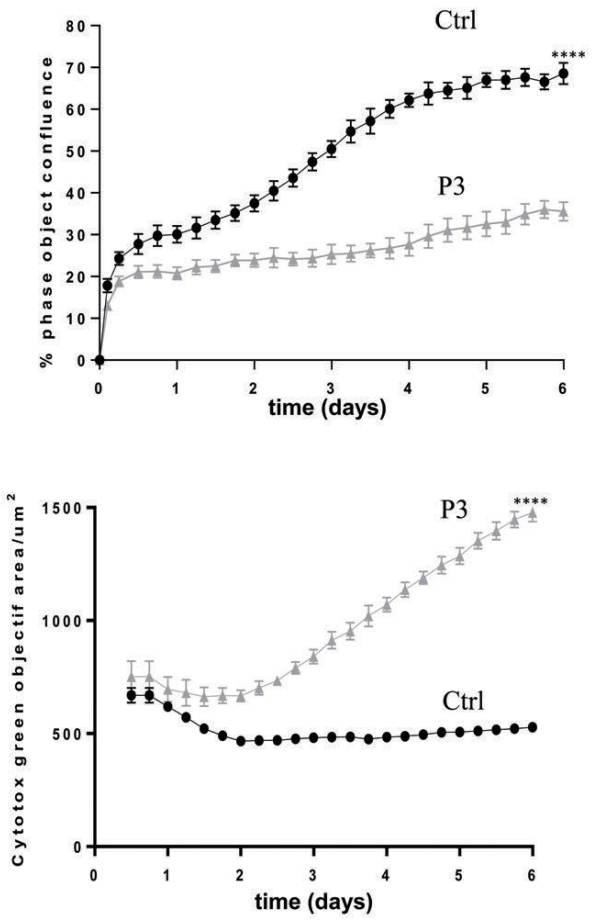

B

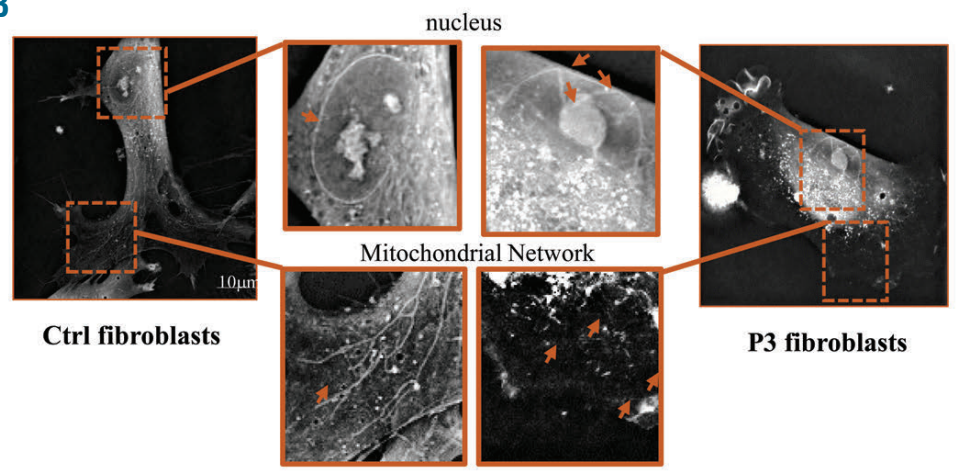

nucleus

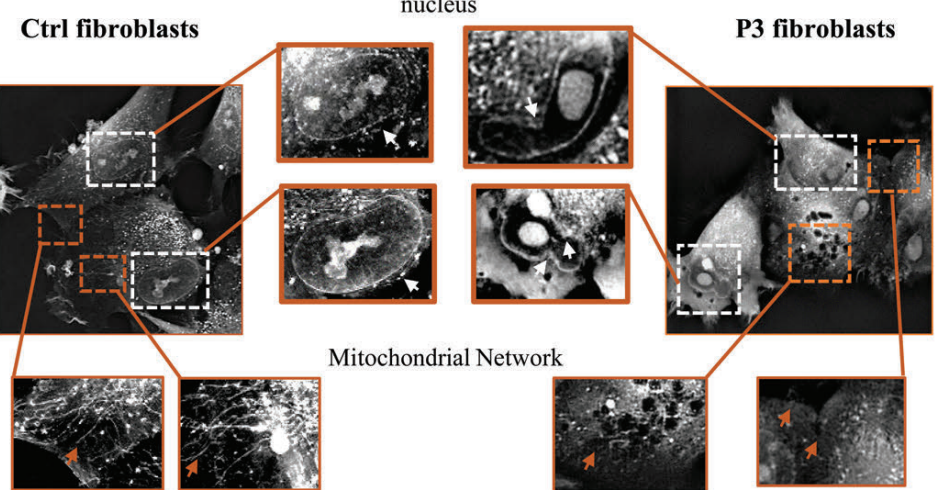

Figure 2. Patient fibroblasts are affected by the G12R mutation. (A) Proliferation kinetics for control fibroblasts (Ctrl) and patient 3's fibroblasts (P3), using the Incucyte assay. The percentage of confluence (\%, upper graph) was measured every 6 hours $(h)$ for 6 days ( $n=3$ wells for both Ctrl and P3). The graph is representative of two independent experiments. Evaluation of cell death (represented as the green fluorescent cells, lower panel) for control (Ctrl) and patient 3's fibroblasts (P3), with the Cytotox green reagent added $6 \mathrm{~h}$ after the start of the culture. Measurements were made every $6 \mathrm{~h}$ for 6 days ( $\mathrm{n}=3 \mathrm{wells}$ for Ctrl and P3), using the Incucyte assay. The graph is representative of two independent experiments. (B) Holotomographic live cell imaging was performed with a 3D Cell Explorer microscope. Cultures of control fibroblasts (left panels) and patient 3's fibroblasts (right panels) are shown. White arrows indicate the nuclear envelope, and brown arrows indicate the mitochondrial network. Scale bar $=10 \mu \mathrm{m}, 1$ pixel= $0.188 \mu \mathrm{m}$. See also Online Supplementary Video M1 and Online Supplementary Figure S2A.

mitochondrial membrane depolarization (quantified as the proportion of "DILC1(5) low" cells) and apoptosis (measured as the proportion of annexin-V-positive cells) were significantly higher than in controls (Figure 3B). It is worthy of note that these alterations were not observed in the GFP- subset (Online Supplementary Figure S1D). Taken as a whole, these findings emphasize the specific correlation between RAC2 mutations at position 12 and impaired cell survival.

As RAC2 is highly expressed in human hematopoietic BM subsets and during human thymopoiesis (Online Supplementary Figure S1E), we transduced HSPC with WPI, WT, G12R or G12V RAC2 cDNAs and induced their differentiation along the granulocyte, monocyte and $\mathrm{T}$ lymphoid lineages, all of which were absent in the three patients. After 7 days of culture with granulocyte colony stimulating factor, the percentage of GFP-expressing cells and the $\mathrm{GFP}^{+} \mathrm{CD} 15^{+} \mathrm{CD} 11 \mathrm{~b}^{+}$neutrophil counts were significantly lower in the G12R and G12V conditions than in the WPI or WT conditions (Figure 3C). The mitochondrial membrane potential and ROS production were also low, and were associated with a high level of apoptosis (Figure 3D). Differentiation towards the monocyte lineage and differentiation in colony-forming unit assays gave similar results (data not shown). Upon exposure to the Notch ligand -like-4 (DL4) culture system (which enables the differen- tiation of HSPC into $\mathrm{CD}^{+} \mathrm{T}$-cell progenitors in 7 days $^{24}$ ), the $\mathrm{GFP}^{+}$subset count in the G12R and G12V conditions was very low (relative to the WT and WPI conditions), and $\mathrm{GFP}^{+} \mathrm{CD}^{+} \mathrm{T}$-cell progenitors were almost completely absent (Figure 3E). Taken as a whole, our results underline: (i) the key function of the GDP/GTP-bound RAC2 balance in the survival and differentiation of the lympho-myeloid compartment; and (ii) the involvement of the RAC2 signaling pathway in HSPC function. We next compared the impact of G12R mutation on HSPC proliferation with that of previously described RAC2 LOF and GOF missense mutations (p.D57N and p.E62K, respectively)..$^{15,16,19}$ During the 8-day culture, the number of GFP+transduced HSPC was significantly lower in G12R conditions and slightly lower in E62K conditions (Online Supplementary Figure S3A and $B$ ). Unlike the G12R mutation, the D57N and E62K mutations had no impact on ROS production or mitochondrial membrane depolarization (Online Supplementary Figure S3C).

Taken as a whole, our observations highlighted the correlation between the high active GTP-bound RAC2 state produced by the G12R mutation and the impairment of HSPC survival and differentiation. These findings fit with the clinical and immunological phenotype observed in our three patients and with the less severe phenotype displayed by patients carrying E62K or D57N mutations. 
A

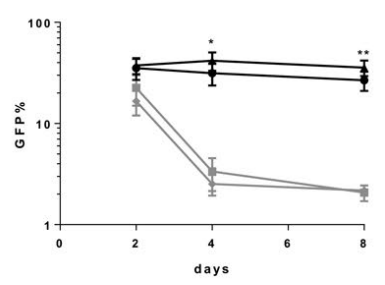

C

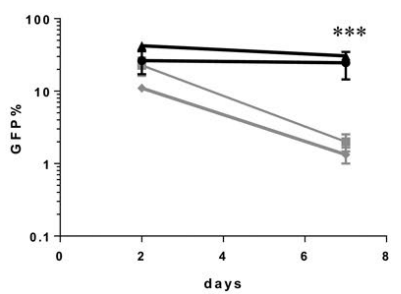

B

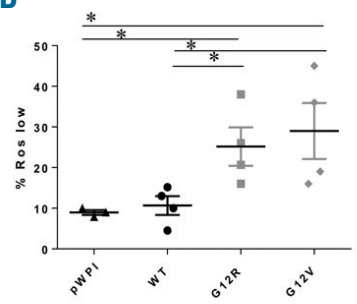

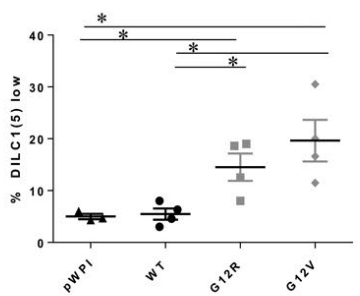

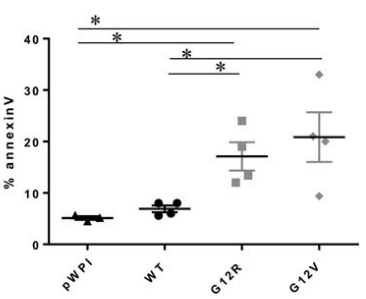

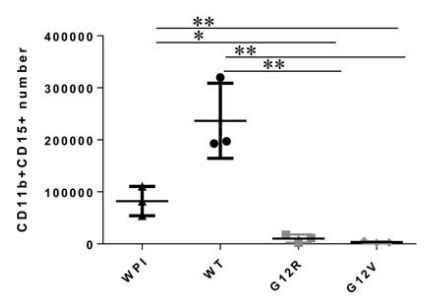

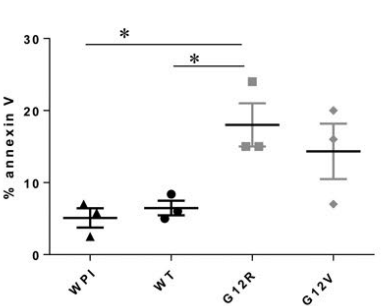

$\mathrm{E}$
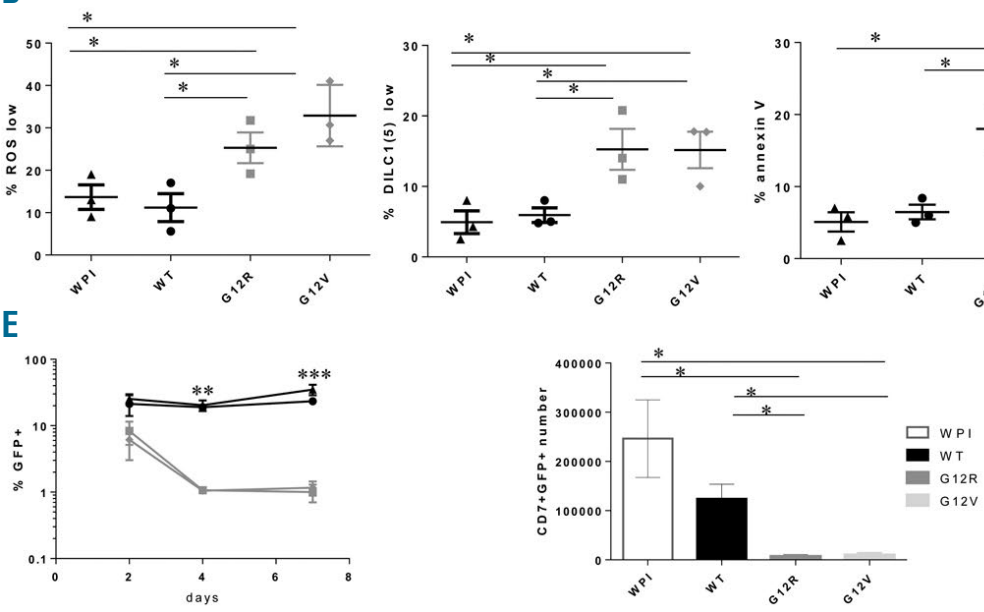

Figure 3. The G12R mutation inhibits hematopoietic stem/progenitor cell (HSPC) proliferation and differentiation. (A) Proliferation of $\mathrm{CD}_{3} 4^{+}$cells in an 8-day culture. Flow cytometry was used to analyze the change over time in the percentage (\%) of GFP-expressing cells $\left(\mathrm{GFP}^{+}\right.$) from day 2 (when the transduction efficiency was measured) until day 8 (the tion efficiency was measured) until day 8 (the end of the culture). (B) The proportions of "ROS low", "DILC1(5) low" and annexin-V-positive $\mathrm{GFP}^{+}$live cells were determined on day 4 . All the analyses were performed in the live cell (7-AADnegative) gate. The results are quoted as the mean \pm standard error of mean (SEM) of four independent experiments. (C) Neutrophil differentiation in a 7-day culture. Flow cytometry was used to analyze the change over time in the percentage (\%) of $\mathrm{GFP}^{+}$cells from day 2 (when the transduction efficiency is measured) until day 8 (end of the culture) and the number of (end of the culture) and the number of cytes $\left(\mathrm{CD} 11 \mathrm{~b}^{+} \mathrm{CD} 15^{+}\right)$among the GFP+ live cells (D) The proportions of "ROS low", "DILC1(5) low" and annexin-V-positive cells among the $\mathrm{GFP}^{+}$live cells were analyzed on day 4 . Results are quoted as the mean \pm SEM of three independent experiments. (E) T-cell differentiation in a 7-day culture $(n=3)$. Flow cytometry was used to analyze the change over time in the percent age (\%) of GFP+ from day 2 (when the tranduction efficiency is measured) until day 7 (end of tion efficiency is measured) until day 7 (end of the culture). The number of T-cell progenitors $\left(C D 7^{+}\right)$was evaluated by flow cytometry on day 7 in the GFP ${ }^{+}$live cell gate (7-AAD-negative). The results are quoted as the mean $\pm S E M$ of three independent experiments. ${ }^{*} P<0.05 ; * * P<0.01$ $* * * P<0.001$. WT: wild-type.

\section{Discussion}

In three SCID patients with innate and adaptive immune system defects, we identified a heterozygous, dominant missense mutation (p.G12R) in the highly conserved GDP/GTP binding domain of the RAC2 protein. Given the severity of the clinical presentation, the patients had to undergo HSCT in the first few weeks of life. The observation of $\mathrm{AD}$ inheritance broadens the clinical spectrum of $\mathrm{RD}$, as we can now distinguish between two forms: a recessive syndromic form associated with deafness and AK2 mutations, and a non-syndromic disease associated with a hypoplastic BM and a specific AD G12R mutation in RAC2. The absence of sensorineural hearing loss in the latter form might be correlated with the predominant expression of RAC2 in hematopoietic lineages. These data fit with the observation whereby in murine models, RAC1 and RAC3 (but not RAC2) are involved in the development of the inner ear; ${ }^{25}$ however, this has yet to be confirmed in human studies. The HSCT performed as soon as possible after birth in two patients with the G12R mutation restored hematopoiesis and highlights the non-redundant regulatory role of RAC2 in this process. In line with this observation, we demonstrated the drastic effect of the G12R RAC2 mutation on cell proliferation and survival, especially in cord blood HSPC. These results are in agreement with previous reports on the role of RAC2 in the regulation of $\mathrm{HSPC}^{10,26}$ and during $\mathrm{T}$-cell dif- ferentiation via the activation of $\mathrm{Wnt} / \beta$-catenin pathways. ${ }^{9}$ Furthermore, the constitutive active form of RAC2 G12V has already been described as preventing thymocyte differentiation and inducing apoptosis in the mouse. ${ }^{27}$ It should be noted that, despite the observation of fibroblast defects in vitro, skin alterations have not been noted for P1 and P2 more than 10 years after HSCT, suggesting that, in vivo, other RHO family members (or perhaps other molecules present in the microenvironment) provide compensatory survival signals to non-hematopoietic cells. The same dichotomy has been reported in $\mathrm{RD}$ : fibroblasts from $\mathrm{RD}$ patients displayed impairments in apoptosis and mitochondrial function in vitro, ${ }^{5}$ whereas the patients having undergone HSCT have not reported any skin or tissue lesions (24 years after HSCT, for the case with the longest follow-up). Taken as a whole, these data demonstrate that RAC2 is mandatory at different hematopoietic checkpoints; hence, the unregulated activity of GTP-bound RAC2 driven by the G12R mutation might explain the severe BM hypoplasia and the absence of circulating leukocytes in P1, P2, and P3.

In HSPC, RHO GTPases also regulate cell trafficking and ROS production. ${ }^{9,26}$ In particular, the literature data show that RAC2 mutant cells are associated with unbalanced actin cycling and hyper-segmented nuclei, ${ }^{15,16,18,19,28}$ two features associated with defective cytokinesis, a process involving RHO GTPases. ${ }^{29-31}$ The highly segmented nuclear membranes found in P3's fibroblasts are consistent 
with these data and suggest that the organization of the cytoskeleton is altered in these cells. However, the mutation described herein could not be classified as an actinopathy such as Wiskott-Aldrich syndrome, ${ }^{32}$ Dock8 deficiency $^{33}$ or warts, hypogammaglobulinemia, infections, and myelokathexis (WHIM) syndrome. ${ }^{34}$ Although these diseases are characterized by impaired leukocyte migration, autoimmunity, and/or malignancies, none is associated with BM hypoplasia or a severe SCID phenotype. Therefore, our present observation is the first to have evidenced cytokinesis failure in SCID patients.

In cell lines, actin dynamics have been described as a key regulator of the mitochondrial network, ${ }^{35}$ suggesting that the mitochondrial defects observed in the G12R mutated HSPC might be a consequence of actin dysregulation. In patients bearing D57N or E62K Rac2 mutations, the fact that actin cycling was disrupted in neutrophils ${ }^{15,19}$ but that mitochondrial membrane depolarization was not evident in the HSPC challenges this argument and suggests that RAC2 could regulate mitochondrial integrity and function through an actin-independent mechanism. This assumption fits with previous reports demonstrating that: (i) RAC2 interacts with SAM50, a mitochondrial transporter involved in the regulation of mitochondrial membrane potential and ROS production: ${ }^{36,37}$ (ii) unregulated RAC2 activation disrupts ROS production, $28,88,39$ which is required for the regulation of mitochondrial dynamics. ${ }^{40}$ Moreover, it is now well established that mitochondrial activity is a key regulator of HSPC homeostasis and functions, ${ }^{41,42}$ suggesting that the inappropriate RAC2 activation driven by G12 mutations directly affects mitochondria and ROS production.

The RAC2 signaling pathways targeted by the G12R mutation are not reported here; however, given that G12R and G12V mutations have the same impact on HSPC, we can reasonably assume that they have the same downstream targets. This assumption is reinforced by the observations in KRAS mutants; both G12R and G12V mutations impaired GTP hydrolysis, ${ }^{22}$ suggesting that the mutant proteins are catalytically inactive. Interestingly, the G12V GOF RAC2 mutation constitutively activates the phospholipases C (PLC) isoforms PLC $\beta 2$ and PLC $\gamma .{ }^{23,43}$ These two PLC interact with phosphatidylinositol 3 kinase (PI3K) and protein kinase $\mathrm{C}$ (PKC), both of which are involved in various signaling pathways (including the ones that regulate HSPC homeostasis). ${ }^{44,45}$

The comparison of RAC2 mutants also highlighted the complexity of an unbalanced RAC2 network, as the D57N and E62K variants had a limited impact on HSPC. This finding might be related to the position of the amino-acid substitution, i.e., inside the GDP/GTP binding pocket (G12R) or in the G3 (D57N) or switch II (E62K) domains (Online Supplementary Figure S1A). In particular, the replacement of glutamate by a lysine at position 62 may impact the GTP bound RAC2 form stability and thus explain the absence of a GTP-bound RAC2 form and a lower level of RAC2 expression, relative to the $\mathrm{G} 12 \mathrm{R}$ condition (Online Supplementary Figure S3D). These findings differ from the report of Hsu et al., ${ }^{19}$ probably because we did not use the same cells or the same techniques to measure RAC2 GTP activity. However, our conclusions fit with the patients' clinical phenotype; we can reasonably assume that the highly active, constitutive GTP-bound form of RAC2 driven by the G12R mutation leads to BM failure and a SCID phenotype. In contrast, the transient overactivation of the RAC2-GTP form driven by the E62K RAC2 mutation is associated with lymphopenia ${ }^{19}$ but not with BM abnormalities. ${ }^{46}$ Taken as a whole, our findings emphasize that various RAC2 mutations differ in their effects on HSPC. These differences may account for the broad observed spectrum of clinical phenotypes, ranging from neutrophil defects to a severe form of SCID. This type of heterogeneity has already been reported for RAG1 mutations involved in common variable immunodeficiency or SCID phenotype. ${ }^{47}$

In summary, the p.G12R RAC2 mutation has a drastic impact on the homeostatic regulation of hematopoiesis, which explains the severity of the patients' clinical and immunological phenotypes. To the best of our knowledge, the present study is the first to have described an AD form of a SCID. Physicians should consider RAC2 gene sequencing for patients with SCID and a clinical presentation of $\mathrm{RD}$. This gene should also be included in newborn screening programs for SCID detection. Finally, future research should seek to characterize the RAC2 signaling pathway and novel downstream targets specifically involved in hematopoietic cell commitment.

\section{Disclosures}

No conflicts of interests to disclose.

\section{Contributions}

CLP and MC supervised and designed the project, CLP analyzed and interpreted data and drafted the manuscript; $A O$ and CT performed the biochemical studies and drafted part of the manuscript; HS, AG and CDS performed some experiments and analyzed data; $P R$ and $Y C$ performed the protein homology modelling and drafted part of the manuscript; AF, DM, CP, JLC and MC provided patient care; CP, JLC, IA and MC helped to draft the manuscript.

\section{Acknowledgments}

We especially thank Prof. Stephane Blanche and the nursing staff of the Department of Pediatric Immunology, Hematology and Rheumatology at Necker-Enfants Malades Hospital for patient care. We also thank Gisèle Froment, Didier Nègre, and Caroline Costa for lentiviral vector production (UMS3444/US8, BioSciences Gerland, Lyon), Olivier Pelle for cell sorting (at the flow cytometry core facility, SFR Necker, Paris), Thibault Courtheoux for the $3 D$ cell imager analysis (Nanolive SA), Myriam Chouteau for technical assistance, and Patrick Revy (from the Genome Dynamics in the Immune System group, Imagine Institute, Paris) for fruitful discussions.

\section{Funding}

This study was funded by the French National Institute of Health and Medical Research (INSERM). The work at the CRCT was funded by an "Equipe Labellisée par la Fondation pour la Recherche Médicale" grant. 


\section{References}

1. Cavazzana M, Bushman FD, Miccio A, André-Schmutz I, Six E. Gene therapy targeting haematopoietic stem cells for inherited diseases: progress and challenges. Nat Rev Drug Discov. 2019;18(6):447-462.

2. Fischer A, Notarangelo LD, Neven B, Cavazzana M. Puck JM. Severe combined immunodeficiencies and related disorders. Nat Rev Dis Primers. 2015;1(1):15061.

3. Picard C, Bobby Gaspar H, Al-Herz W, et al. International Union of Immunological Societies: 2017 Primary Immunodeficiency Diseases Committee Report on Inborn Errors of Immunity. J Clin Immunol. 2018; 38(1):96-128.

4. de Vaal O, Seynhaeve V. Reticular dysgenesia. Lancet. 1959;2(7112):1123-1125.

5. Pannicke U, Hönig M, Hess I, et al. Reticular dysgenesis (aleukocytosis) is caused by mutations in the gene encoding mitochondrial adenylate kinase 2. Nat Genet. 2009;41(1):101-105.

6. Lagresle-Peyrou C, Six EM, Picard C, et al. Human adenylate kinase 2 deficiency causes a profound hematopoietic defect associated with sensorineural deafness. Nat Genet. 2009;41(1):106-111.

7. Six E, Lagresle-Peyrou C, Susini S, et al. AK2 deficiency compromises the mitochondrial energy metabolism required for differentiation of human neutrophil and lymphoid lineages. Cell Death Dis. 2015;6:e1856.

8. Troeger A, Williams DA. Hematopoieticspecific Rho GTPases Rac2 and $\mathrm{RhoH}$ and human blood disorders. Exp Cell Res. 2013; 319(15):2375-2383

9. Nayak RC, Chang K-H, Vaitinadin N-S, Cancelas JA. Rho GTPases control specific cytoskeleton-dependent functions of hematopoietic stem cells. Immunol Rev. 2013;256(1):255-268

10. Gu Y, Filippi M-D, Cancelas JA, et al. Hematopoietic cell regulation by Rac1 and Rac2 guanosine triphosphatases. Science. 2003;302(5644):445-449.

11. Shirsat NV, Pignolo RJ, Kreider BL, Rovera G. A member of the ras gene superfamily is expressed specifically in T, B and myeloid hemopoietic cells. Oncogene. 1990;5(5):769772 .

12. Gu Y, Byrne MC, Paranavitana NC, et al. Rac2, a hematopoiesis-specific Rho GTPase, specifically regulates mast cell protease gene expression in bone marrowderived mast cells. Mol Cell Biol. 2002;22(21):7645-7657.

13. André-Schmutz I, Le Deist F, Hacein-BeyAbina S, et al. Immune reconstitution without graft-versus-host disease after haemopoietic stem-cell transplantation: a phase 1/2 study. Lancet. 2002; 360(9327): 130-137.

14. Olson MF. Rho GTPases, their post-translational modifications, disease-associated mutations and pharmacological inhibitors. Small GTPases. 2018;9(3):203-215.

15. Ambruso DR, Knall C, Abell AN, et al. Human neutrophil immunodeficiency syndrome is associated with an inhibitory Rac2 mutation. Proc Natl Acad Sci U S A. 2000; 97(9):4654-4659.

16. Accetta D, Syverson G, Bonacci B, et al.
Human phagocyte defect caused by a Rac2 mutation detected by means of neonatal screening for T-cell lymphopenia. J Allergy Clin Immunol. 2011;127(2):535-538.e1-2.

17. Alkhairy OK, Rezaei N, Graham RR, et al. RAC2 loss-of-function mutation in 2 siblings with characteristics of common variable immunodeficiency. J Allergy Clin Immunol. 2015;135(5):1380-1384.e1-5.

18. Lougaris V, Chou J, Beano A, et al. A monoallelic activating mutation in RAC2 resulting in a combined immunodeficiency. J Allergy Clin Immunol. 2019;143(4):16491653 .e3.

19. Hsu AP, Donkó A, Arrington ME, et al. Dominant activating RAC2 mutation with lymphopenia, immunodeficiency, and cytoskeletal defects. Blood. 2019; 133(18):1977-1988.

20. Sharapova SO, Haapaniemi E, Sakovich IS, et al. Heterozygous activating mutation in RAC2 causes infantile-onset combined immunodeficiency with susceptibility to viral infections. Clin Immunol. 2019;205:1-5.

21. Scheffzek K, Stephan I, Jensen ON, Illenberger D, Gierschik P. The Rac-RhoGDI complex and the structural basis for the regulation of Rho proteins by RhoGDI. Nat Struct Biol. 2000;7(2):122-126.

22. Hunter JC, Manandhar A, Carrasco MA, Gurbani D, Gondi S, Westover KD. Biochemical and structural analysis of common cancer-associated KRAS mutations. Mol Cancer Res. 2015;13(9):1325-1335.

23. Illenberger D, Walliser C, Strobel J, et al Rac2 regulation of phospholipase C-beta 2 activity and mode of membrane interactions in intact cells. I Biol Chem. 2003;278(10):8645-8652.

24. Reimann C, Six E, Dal-Cortivo L, et al Human T-lymphoid progenitors generated in a feeder-cell-Free delta-like-4 culture system promote T-cell reconstitution in NOD/SCID $/ \mathrm{\gamma c}^{-/-}$Mice. Stem Cells. 2012; 30(8):1771-1780

25. Grimsley-Myers CM, Sipe CW, Wu DK, Lu $X$. Redundant functions of Rac GTPases in inner ear morphogenesis. Dev Biol. 2012; 362(2):172-186.

26. Mulloy JC, Cancelas JA, Filippi M-D, Kalfa TA, Guo F, Zheng Y. Rho GTPases in hematopoiesis and hemopathies. Blood. 2010;115(5):936-947.

27. Lorès $\mathrm{P}$, Morin L, Luna R, Gacon G Enhanced apoptosis in the thymus of transgenic mice expressing constitutively activated forms of human Rac2GTPase. Oncogene. 1997;15(5):601-605

28. Tao W, Filippi M-D, Bailey JR, et al. The TRQOKRP motif located near the C-terminus of Rac2 is essential for Rac2 biologic functions and intracellular localization. Blood. 2002;100(5):1679-1688.

29. Canman JC, Lewellyn L, Laband K, et al. Inhibition of Rac by the GAP activity of centralspindlin is essential for cytokinesis. Science. 2008;322(5907):1543-1546.

30. Normand G, King RW. Understanding cytokinesis failure. Adv Exp Med Biol. 2010; 676:27-55.

31. Verma V, Mogilner A, Maresca TJ. Classical and emerging regulatory mechanisms of cytokinesis in animal cells. Biology (Basel). 2019;8(3)

32. Moulding DA, Moeendarbary E, Valon L,
Record J, Charras GT, Thrasher AJ. Excess Factin mechanically impedes mitosis leading to cytokinesis failure in X-linked neutropenia by exceeding Aurora B kinase error correction capacity. Blood. 2012;120(18):38033811

33. Zhang Q, Dove CG, Hor JL, et al. DOCK8 regulates lymphocyte shape integrity for skin antiviral immunity. J Exp Med. 2014; 211(13):2549-2566.

34. Al Ustwani O, Kurzrock R, Wetzler M Genetics on a WHIM. Br J Haematol. 2014 164(1):15-23.

35. Moore AS, Wong YC, Simpson CL Holzbaur ELF. Dynamic actin cycling through mitochondrial subpopulations locally regulates the fission-fusion balance within mitochondrial networks. Nat Commun. 2016;7:12886.

36. Capala ME, Maat H, Bonardi F, et al Mitochondrial dysfunction in human leukemic stem/progenitor cells upon loss of RAC2. PLoS One. 2015:10(5):e0128585.

37. Capala ME, Pruis M, Vellenga E, Schuringa JJ. Depletion of SAM50 specifically targets BCR-ABL-expressing leukemic stem and progenitor cells by interfering with mitochondrial functions. Stem Cells Dev. 2016 25(5):427-437.

38. Pei H, Zhang J, Nie J, et al. RAC2-P38 MAPK-dependent NADPH oxidase activity is associated with the resistance of quiescent cells to ionizing radiation. Cell Cycle. 2017; 16(1):113-122

39. Nieborowska-Skorska M, Kopinski PK, Ray $\mathrm{R}$, et al. Rac2-MRC-cIII-generated ROS cause genomic instability in chronic myeloid leukemia stem cells and primitive progenitors. Blood. 2012;119(18):4253-4263

40. Ježek J, Cooper KF, Strich R. Reactive oxygen species and mitochondrial dynamics: the yin and yang of mitochondrial dysfunction and cancer progression. Antioxidants (Basel). 2018;7(1).

41. Bigarella CL, Liang R, Ghaffari S. Stem cells and the impact of ROS signaling. Development. 2014:141(22):4206-4218.

42. Ansó E, Weinberg SE, Diebold LP, et al. The mitochondrial respiratory chain is essential for haematopoietic stem cell function. Nat Cell Biol. 2017;19(6):614-625

43. Bunney TD, Opaleye O, Roe SM, et al. Structural insights into formation of an active signaling complex between Rac and phospholipase C gamma 2. Mol Cell. 2009, 34(2):223-233

44. Edling CE, Pedersen M, Carlsson L, Rönnstrand L, Palmer RH, Hallberg B. Haematopoietic progenitor cells utilise conventional PKC to suppress PKB/Akt activity in response to c-Kit stimulation. $\mathrm{Br}$ Haematol. 2007;136(2):260-268.

45. Elich M, Sauer K. Regulation of hematopoietic cell development and function through phosphoinositides. Front Immunol. 2018; 9:931.

46. Smits BM, Lelieveld PHC, Ververs FA, et al A dominant activating RAC2 variant associated with immunodeficiency and pulmonary disease. Clin Immunol. 2019: 108248 .

47. Notarangelo LD, Kim M-S, Walter JE, Lee YN. Human RAG mutations: biochemistry and clinical implications. Nat Rev Immunol. 2016;16(4):234-246 